\title{
PARÁMETROS SUBJETIVOS DE SUEÑO Y ESTADO DE ÁNIMO DISFÓRICO
}

\author{
M. CARMEN CANO ${ }^{1}$, ELENA MIRÓ ${ }^{2}$, LOURDES ESPINOSA-FERNÁNDEZ ${ }^{1}$ \\ y GUALBERTO BUELA-CASAL ${ }^{2}$ \\ ' Universidad de Jaén \\ ${ }^{2}$ Universidad de Granada
}

(Aceptado en diciembre de 2003)

El objetivo del presente estudio es analizar la relación entre diversos parámetros subjetivos de cantidad y calidad de sueño y estado de ánimo disfórico en una muestra de sujetos sanos bajo condiciones normales. La muestra estaba compuesta por 257 estudiantes universitarios. Se administró un cuestionario sobre hábitos de sueño y el Inventario de Depresión de Beck. Se han encontrado diferencias significativas en estado de ánimo disfórico en función de las horas habituales de sueño pero no de las horas necesarias de sueño. Los sujetos que duermen habitualmente menos de 7 horas presentan un estado de ánimo más disfórico. También se han encontrado diferencias en estado de ánimo disfórico en función del ajuste o no entre horas habituales y horas necesarias de sueño. Los sujetos que duermen habitualmente menos horas de las que necesitan muestran más síntomas de disforia. Por otro lado, el estado de ánimo disfórico se relaciona con latencia de sueño, número de despertares nocturnos, regularidad del sueño y grado de satisfacción con la calidad de sueño. Los datos apoyan la importancia del sueño y su relación con medidas del bienestar psicológico en poblaciones no clínicas.

Palabras Clave: Cantidad de sueño, calidad de sueño, estado de ánimo disfórico.

\section{Subjective parameters of sleep and dysphoric mood}

The objective of the present study is to analyse the relationship between several subjective parameters of sleep quantity and sleep quality and dysphoric mood in healthy subjects under normal conditions. The sample was composed of 257 university students. A questionnaire about sleep habits and the Beck Depression Inventory were carried out. There were significant differences in dysphoric mood according to habitual sleep duration but not to necessary sleep duration. The subjects who habitually sleep for less than 7 hours are those who present a worse dysphoric mood. There were differences in dysphoric mood dependent upon the existence or otherwise of an adjustment between habitual and necessary sleep hours. The subjects who habitually sleep less hours than they need are those who show more symptoms of dysphoric mood. On the other hand, it has been found that dysphoric mood is related to sleep latency, number of awakenings, sleep regularity and satisfaction with sleep quality. The data supports the importance of sleep and its relationship to measures of well-being in the nonclinical population.

Key Words: Sleep quantity, sleep quality, dysphoric mood.

\section{INTRODUCCIÓN}

\section{Son diversas las líneas de investigación} que han constatado la estrecha relación

Correspondencia: $\mathrm{M}$. del Carmen Cano Lozano, Departamento de Psicología, Universidad de Jaén. Paraje Las Lagunillas s/n, Edific. D-2, 23071 Jaén, España. Teléfono: 953011 990, Fax: 953012197. Correo-e: mccano@ujaen.es existente entre sueño y bienestar psicológico. En muestras clínicas, esta relación ha sido ampliamente estudiada. Se sabe que las alteraciones del sueño están presentes en diversos trastornos psicopatológicos: trastornos del estado de ánimo, trastornos de ansiedad, trastornos de la personalidad, trastornos alimenticios, esquizofrenia, etc. (Benca, Obermeyer, 
Thisted y Gillin, 1992). De todos estos trastornos psicopatológicos el más vinculado a las alteraciones del sueño y el que mayor número de estudios ha generado ha sido la depresión. Aproximadamente, el $80 \%$ de los pacientes con depresión se quejan de un deterioro tanto en la cantidad como en la calidad de su sueño (Cano, Espinosa, Miró y BuelaCasal, 2003; Dew et al., 1996; Gillin et al., 1984; Kupfer, 1995; Riemann, Berger y Voderholzer, 2001). Desde otra perspectiva, diversos estudios han establecido que la desviación por encima o por debajo del patrón intermedio de sueño (sujetos que duermen entre 7 y 8 horas cada día) puede aumentar el riesgo de padecer trastornos psicopatológicos. Breslau, Roth, Rosenthal y Andreski (1997) encuentran que las personas con patrón de sueño corto (aquellas que duermen 6 horas o menos cada día) o patrón de sueño largo (aquellas que duermen 9 horas o más cada día), mostraban una mayor prevalencia de depresión, ansiedad y trastornos relacionados con el abuso de sustancias. Las ratios para la depresión eran mucho más altas que para los otros trastornos. También encuentran que el riesgo de depresión era casi cuatro veces más alto cuando había historia previa de sueno insuficiente o dificultades para dormirse que cuando el patrón de sueño era intermedio o incluso largo. Otros estudios sí han encontrado asociaciones entre la presencia de patrón de sueño largo y el riesgo de sufrir depresión posteriormente (Ford y Kamerow, 1989).

En muestras no clínicas, la mayor parte de los estudios se han centrado en analizar los efectos de diferentes manipulaciones del sueño como privación de sueño, reducción de sueño o cambios en el horario de sueño, en la salud física y psicológica. Generalmente estos estudios coinciden en señalar que este tipo de manipulaciones del sueño suelen producir efectos perjudiciales en un amplio rango de variables asociadas al bienestar psicológico. Por ejemplo, la privación de sueño produce generalmente un decremento en activación, vigor y vigilancia y un incremento en fatiga, somnolencia, ansiedad, confusión e irritabilidad (Brendel et al., 1990; Cutler y Cohen, 1979; Gillberg y Akerstedt, 1981; Hill, Welch y Godfrey, 1996; Leproult et al., 2003; Mikulincer, Babkoff, Caspy y Sing, 1989; Miró, Cano y Buela-Casal, 2002a; Reynolds et al., 1986).

Tomados en conjunto estos estudios sugieren estrechas relaciones entre el sueño y el bienestar psicológico. En particular, la duración del sueño, el momento del comienzo del sueño, la calidad del sueño, despertares nocturnos y latencia de sueño MOR (con movimientos oculares rápidos) han sido variables asociadas con cambios en el estado de ánimo (Totterdell, Reynolds, Parkinson y Briner, 1994). Sin embargo, estas asociaciones han sido encontradas en condiciones anómalas y extremas tales como restricciones de sueño, condiciones particulares de trabajo y desórdenes clínicos. Han sido pocos los estudios que se han ocupado de la relación entre sueño y estado de ánimo en sujetos sanos bajo condiciones normales. Para una mejor comprensión de la relación entre sueño y bienestar psicológico se hace necesario estudiar los hábitos de sueño sin la influencia directa de alteraciones clínicas o situaciones restrictivas.

En este sentido, se ha demostrado que la duración del sueño puede influir en una amplia variedad de aspectos físicos y psicológicos (Miró, Iáñez y Cano, 2002c). Kripke, Garfinkel, Wingard, Klauber y Marter (2002) han publicado una reevaluación de los datos de su estudio de 1979 que incluyó a más de un millón de personas de entre 30 y 102 años. Los datos señalan que las personas que dormían habitualmente unas 7 horas tuvieron los mejores índices de supervivencia. 
En cambio, dormir menos de 4 horas o más de 8 horas aumenta el riesgo de muerte temprana. Kojima et al. (2000) han analizado también la relación entre la mortalidad y los patrones de sueño teniendo en cuenta no sólo la duración del sueño sino también la calidad del sueño. Un total de 5.322 japoneses de entre 20 y 67 años completaron un cuestionario de salud y estilos de vida que incluía los patrones de sueño. Se observó una relación entre los diferentes patrones de sueño y la tasa de mortalidad total. En los varones que tenían un patrón de sueño corto o largo, frente a aquellos que dormían un promedio de 7-8 horas, se evidenciaba un incremento en el riesgo de mortalidad. Esta relación no se encontró en el caso de las mujeres. Otros estudios han llegado a las mismas conclusiones utilizando muestras de sujetos ancianos (Bazargan, 1996; Habte-Gabr et al., 1991) y muestras de sujetos adolescentes (Mahon, 1995). En el estudio de Duncan, Bomar, Nicholson y Wilson (1995) el ejercicio moderado y los hábitos regulares de sueño (7-8 horas) eran predictores de mejor salud mental en una muestra de 490 universitarios. Otros estudios, sin embargo, no han encontrado relación entre duración del sueño y bienestar psicológico (Johns, Bruce y Masterton, 1974; Spinweber, Johson y Chin, 1985; Webb, 1979). Pilcher, Ginter y Sadowsky (1997) analizan en dos estudios la relación entre cantidad de sueño y medidas de salud y bienestar psicológico. En un estudio encuentran que la cantidad de sueño no correlacionaba significativamente con ninguna medida de salud o bienestar psicológico y en el otro estudio sólo dos medidas del bienestar psicológico, fatiga y confusión, correlacionaban negativamente con la cantidad de sueño.

Diversos estudios sugieren también la existencia de una relación positiva entre calidad de sueño y salud autoinformada
(Hyyppa, Kronholm y Mattlar, 1991; Lugaresi et al., 1983). Oullet (1995) efectuó una investigación cuyo objetivo es identificar los factores asociados con la satisfacción del sueño en ancianos. La muestra la integraban 130 personas mayores de 65 años que no sufrían de ningún tipo de enfermedad. Encuentran que los principales factores que contribuían positivamente a la satisfacción del sueño eran la cantidad, la calidad y la profundidad del sueño y, de forma negativa, los despertares nocturnos. A su vez, a mayor satisfacción de sueño autoevaluada menores síntomas de depresión y ansiedad. De estos aspectos, era la depresión la que mejor correlacionaba con la satisfacción del sueño.

Estudios que hayan analizado conjuntamente la influencia de los dos parámetros de sueño (cantidad y calidad de sueño) en el bienestar psicológico son muy escasos. En el estudio llevado a cabo por Pilcher et al. (1997) se encuentra que las medidas de salud y bienestar se relacionan más con parámetros de calidad que con parámetros de cantidad de sueño. Pobre calidad de sueño correlacionaba significativamente con mayor número de quejas físicas y con un incremento de tensión, depresión, ira, fatiga y confusión.

Con objeto de profundizar más en la relación entre sueño y bienestar psicológico, en el presente estudio se analiza la relación entre diferentes parámetros subjetivos de cantidad y calidad de sueño y estado de ánimo disfórico en una muestra de sujetos sanos bajo condiciones normales.

\section{MÉTODO}

El método para llevar a cabo el estudio es el cuasiexperimental. Para ello se siguieron las directrices planteadas por Bobenrieth (2002) y Montero y León (2002). 


\section{Sujetos}

La muestra estaba compuesta por 257 estudiantes universitarios sanos (177 mujeres y 80 hombres) con edades comprendidas entre los 18 y 30 años (media $=21,48 ;$ desviación típica $=2,12$ ). Para seleccionar a los sujetos se utilizó un cuestionario elaborado para tal fin en el que se exploraba el estado de salud física y psicológica, el consumo de fármacos, tabaco, alcohol y otras drogas, la ingesta de café, té u otros estimulantes y la posible existencia de trastornos del sueño. Los criterios de selección utilizados fueron no padecer ninguna enfermedad médica o psiquiátrica pasada o presente con especial atención a los antecedentes o presencia de depresión, no estar tomando ningún tipo de medicación o droga, no ingerir más de 3 tazas de café al día u otros estimulantes y no presentar o haber presentado trastornos del sueño como insomnio o hipersomnia.

\section{Instrumentos}

\section{-Variables de sueño}

Se aplicó un cuestionario sobre hábitos de sueño que contenía información de los siguientes aspectos: (1) duración habitual del sueño (en horas), (2) duración necesaria del sueño (en horas), (3) latencia de sueño (menos de 15 minutos, entre 15-30 minutos, entre 30-60 minutos y más de 1 hora), (4) número de despertares nocturnos (raramente o nunca, una vez por noche y varias veces en la noche), (5) profundidad del sueño (ligero, medio, profundo), (6) regularidad del sueño (regular, irregular) y (7) satisfacción con calidad de sueño (si-no). Para contestar el cuestionario se pedía a los sujetos que basaran su respuesta en lo que era su pauta habitual de sueño y no en días aislados. El cuestionario de sueño proporciona 2 estima- ciones de la cantidad de sueño (cuestiones 1 y 2) y 7 estimaciones de la calidad de sueño (cuestiones 3 a 7).

La estimación autoinformada de sueño se ha encontrado que correlaciuna de forma elevada $(r=0,50)$ con medidas polisomnográficas de sueño (Matousek, Nuth y Petersen, 1983). Además, los datos de autoinforme proporcionan información adicional acerca de los efectos de las alteraciones psicológicas sobre el patrón de sueño no proporcionada por medidas objetivas (Shaver, Giblin y Paulsen, 1991).

—Estado de ánimo disfórico

El estado de ánimo disfórico se evaluó mediante el Inventario de Depresión de Beck (Beck Depression Inventory, BDI; Beck, Rush, Shaw y Emery, 1979) (versión de Vázquez y Sanz, 1991). Se utilizó la forma completa de 21 ítems excluyendo la puntuación del ítem 16 referido a dificultades del sueño. Este tipo de prueba fue seleccionada no sólo por sus avaladas propiedades psicométricas (Beck, Steer y Garbin, 1988; Steer, Beck y Garrison, 1986) sino por la posibilidad de aplicar este instrumento en poblaciones no clínicas en las que el BDI puede identificar sujetos con un estado de ánimo disfórico (Carroll, Fielding y Blashky, 1973).

\section{Análisis de datos}

En primer lugar se llevaron a cabo análisis descriptivos globales tanto de las variables de sueño como de la variable estado de ánimo disfórico que incluían porcentaje para las variables categóricas y media y desviación típica para las variables continuas. Para determinar las diferencias en estado de ánimo disfórico en función de los diferentes indicadores subjetivos de sueño se llevaron a cabo pruebas no paramétricas, la $\mathrm{H}$ de Krus- 
kall-Wallis para $\mathrm{K}$ muestras independientes y la U de Mann-Whitney para dos muestras independientes.

Para determinar la relación entre horas de sueño y estado de ánimo disfórico se estableció una distinción entre los denominados sujetos con patrón de sueño corto (duermen diariamente una media de 6 horas o menos), los sujetos con patrón de sueño largo (duermen diariamente una media de 9 horas o más) y aquellos con patrón de sueño intermedio (duermen aproximadamente entre 7-8 horas cada día) (Moorcroft, 1993). Asimismo, para los análisis se diferenció entre horas habituales de sueño (determinadas fundamentalmente por condicionantes ambientales), horas necesarias de sueño (patrón verdadero de sueño) y desajuste entre horas habituales de sueño y horas necesarias de sueño (diferencia entre ambos parámetros de sueño). En este último caso se determinaron también tres grupos: sujetos privados de sueño (duermen menos horas de las que necesitan), sujetos con exceso de sueño (duermen más horas de las que necesitan) y sujetos con ajuste de sueño (duermen las horas que necesitan).

\section{RESULTADOS}

\section{Datos descriptivos globales}

Los datos descriptivos de las variables de sueño y del estado de ánimo disfórico en la muestra total, en la muestra de hombres y en la muestra de mujeres se encuentran resumidos en la Tabla 1.

Cantidad de sueño y estado de ánimo disfórico

-Horas habituales de sueño

En cuanto a las horas habituales de sueño los grupos quedaron establecidos de la siguiente manera: 22 sujetos dormían 6 horas o menos, 174 sujetos dormían entre 7 y 8 horas y 61 sujetos dormían 9 horas o más. Los resultados indican que, en la muestra total, existen diferencias significativas $(K-W=5,97$, g.l. $=2, p £$ 0,050 ) en estado de ánimo disfórico en función de las horas habituales de sueño. Comparaciones posteriores indican que las diferencias significativas ( $U=466,00$, $p<0,05)$ se dan entre los dos grupos extremos. Los sujetos que duermen habitualmente 6 horas o menos tienen una puntuación más elevada en el BDI (media $=10,45$; desviación típica $=9,11$ ) que los sujetos que duermen habitualmente 9 horas o más (media $=5,67$; desviación típica $=5,31$ ) (Figura 1a). No se han encontrado diferencias entre hombres y mujeres.

\section{- Horas necesarias de sueño}

En cuanto a las horas necesarias de sueño los grupos quedaron establecidos de la siguiente manera: 10 sujetos con patrón de sueño corto, 144 sujetos con patrón de sueño intermedio y 103 sujetos con patrón de sueño largo. En la muestra total no se han encontrado diferencias significativas $(K-W=4,30$, g.l. $=2, p=$ 0,11 ) en estado de ánimo disfórico en función de las horas necesarias de sueño. En la muestra de mujeres sí se han encontrado diferencias significativas $(K$ $W=6,13$, g.l. $=2, p \leq 0,050)$. Concretamente existen diferencias significativas entre mujeres con patrón de sueño corto y patrón de sueño intermedio ( $U=$ $107,00, p<0,05$ ) y entre mujeres con patrón de sueño corto y patrón de sueño largo $(U=101,50, p<0,05)$. Las puntuaciones más bajas en el BDI corresponden a las mujeres con patrón de sueño corto ( media $=1,67 ;$ desviación típica $=1,63$ ), seguidas de las mujeres con patrón de sueño intermedio (media $=7,48$; desvia- 
Tabla 1. Estadísticos descriptivos de las variables de sueño y del estado de ánimo disfórico

\begin{tabular}{|c|c|c|c|}
\hline \multirow[t]{3}{*}{ Duración habitual de sueño } & Horas & Total & $8,29(1,04)$ \\
\hline & & Hombres & $8,13(1,12)$ \\
\hline & & Mujeres & $8,36(0,99)$ \\
\hline \multirow[t]{3}{*}{ Duración necesaria de sueño } & Horas & Total & $8,39(1,22)$ \\
\hline & & Hombres & $8,09(1,20)$ \\
\hline & & Mujeres & $8,52(1,20)$ \\
\hline \multirow[t]{12}{*}{ Latencia de sueño } & Menos de 15 minutos & Total & $99(39,4 \%)$ \\
\hline & & Hombres & $32(40,5 \%)$ \\
\hline & & Mujeres & $67(38,3 \%)$ \\
\hline & $15-30$ minutos & Total & $109(43,4 \%)$ \\
\hline & & Hombres & $31(39,2 \%)$ \\
\hline & & Mujeres & $81(46,3 \%)$ \\
\hline & $30-60$ minutos & Total & $28(11,1 \%)$ \\
\hline & & Hombres & $11(13,9 \%)$ \\
\hline & & Mujeres & $17(9,7 \%)$ \\
\hline & Más de 1 hora & Total & $15(5,9 \%)$ \\
\hline & & Hombres & $5(6,3 \%)$ \\
\hline & & Mujeres & $10(5,7 \%)$ \\
\hline \multirow[t]{9}{*}{ N. ${ }^{\circ}$ de despertares nocturnos } & Raramente o nunca & Total & $190(74,8 \%)$ \\
\hline & & Hombres & $57(71,3 \%)$ \\
\hline & & Mujeres & $134(75,7 \%)$ \\
\hline & Una vez cada noche & Total & $45(17,7 \%)$ \\
\hline & & Hombres & $17(21,3 \%)$ \\
\hline & & Mujeres & $30(16,9 \%)$ \\
\hline & Varias veces en la noche & Total & $19(7,4 \%)$ \\
\hline & & Hombres & $6(7,5 \%)$ \\
\hline & & Mujeres & $13(7,3 \%)$ \\
\hline \multirow[t]{9}{*}{ Profundidad del sueño } & Ligero & Total & $22(8,9 \%)$ \\
\hline & & Hombres & $5(6,3 \%)$ \\
\hline & & Mujeres & $17(10,1 \%)$ \\
\hline & Medio & Total & $116(47,3 \%)$ \\
\hline & & Hombres & $34(42,5 \%)$ \\
\hline & & Mujeres & $84(50,0 \%)$ \\
\hline & Profundo & Total & $107(43,6 \%)$ \\
\hline & & Hombres & $41(51,3 \%)$ \\
\hline & & Mujeres & $67(39,9 \%)$ \\
\hline \multirow[t]{6}{*}{ Regularidad del sueño } & Regular & Total & $161(83,8 \%)$ \\
\hline & & Hombres & $44(75,9 \%)$ \\
\hline & & Mujeres & $118(86,8 \%)$ \\
\hline & Irregular & Total & $31(16,1 \%)$ \\
\hline & & Hombres & $14(24,1 \%)$ \\
\hline & & Mujeres & $18(13,2 \%)$ \\
\hline \multirow[t]{6}{*}{ Satisfacción con la calidad de sueño } & $\mathrm{Si}$ & Total & $180(71,1 \%)$ \\
\hline & & Hombres & $58(72,5 \%)$ \\
\hline & & Mujeres & $124(70,5 \%)$ \\
\hline & No & Total & $73(28,8 \%)$ \\
\hline & & Hombres & $22(27,5 \%)$ \\
\hline & & Mujeres & $52(29,5 \%)$ \\
\hline \multirow[t]{3}{*}{ Estado de ánimo disfórico } & BDI & Total & $7,38(6,72)$ \\
\hline & & Hombres & $7,31(6,16)$ \\
\hline & & Mujeres & $7,41(6,97)$ \\
\hline
\end{tabular}

Nota: Se muestran las puntuaciones medias y (desviaciones típicas) para las variables duración habitual de sueño, duración necesaria de sueño y estado de ánimo disfórico. En el resto de variables de sueño se muestra el número de sujetos y (porcentaje). 


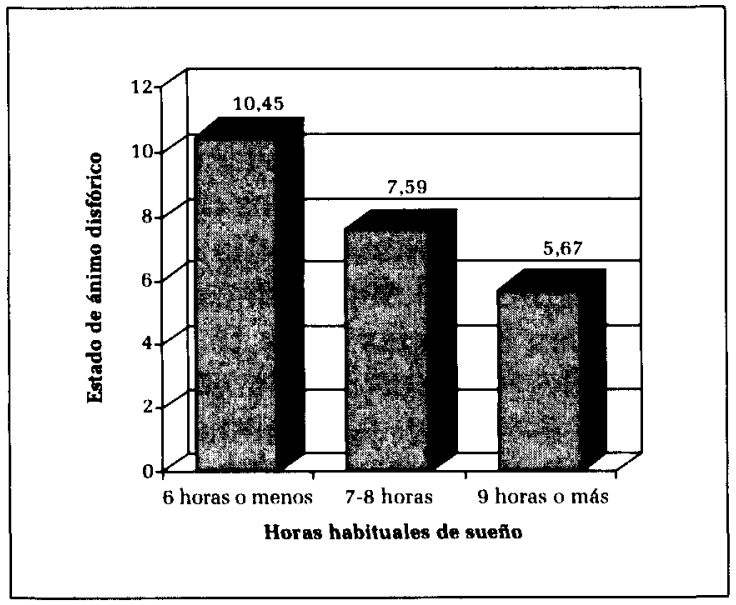

(a)

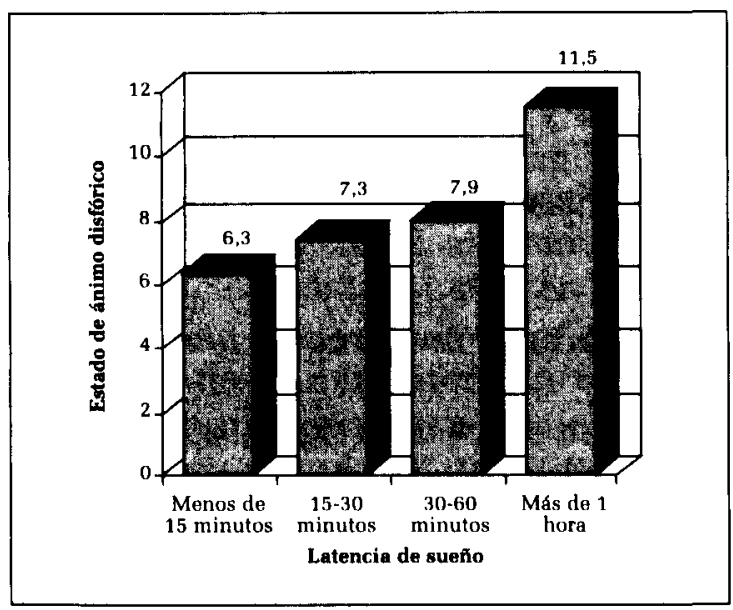

(c)

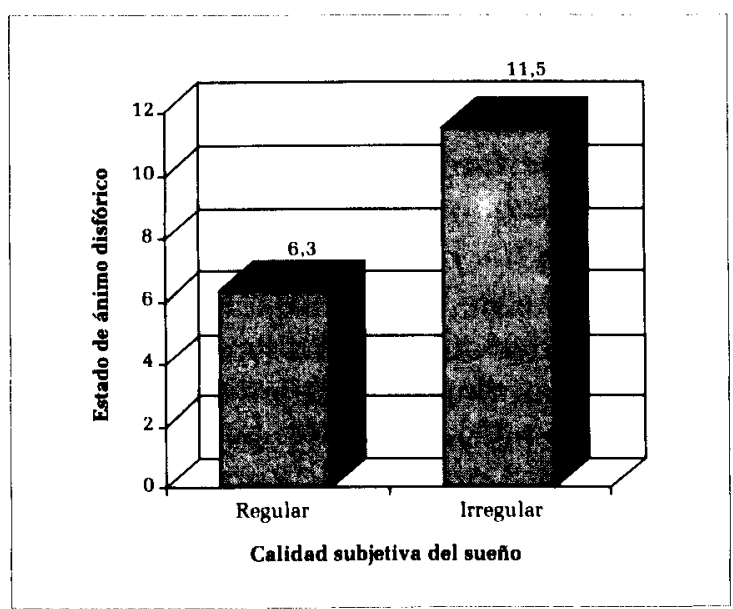

(e)

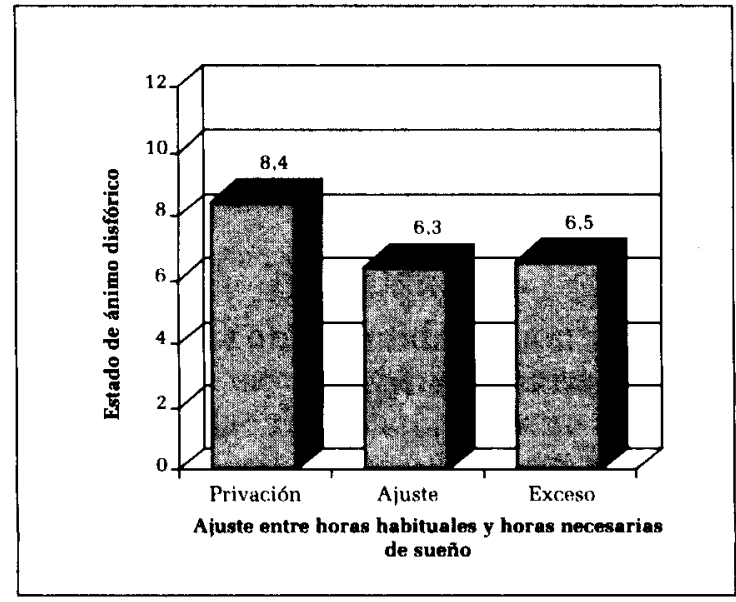

(b)

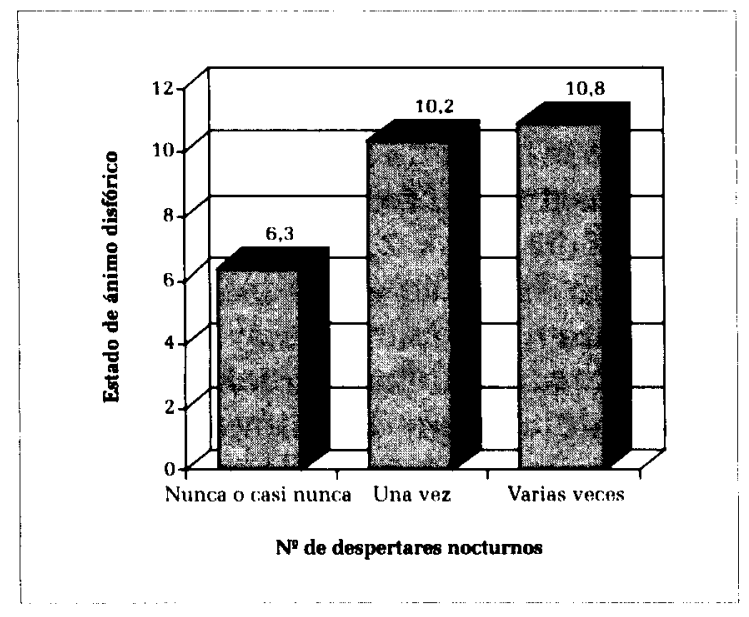

(d)

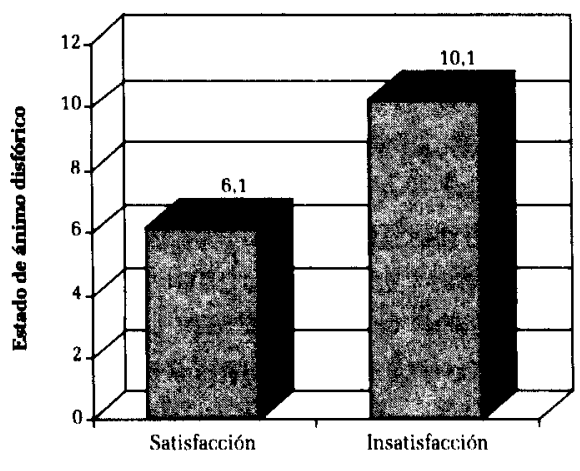

Grado de satisfacción con la calidad del sueño

(f)

Figura 1. Estado de ánimo disfórico en función de las horas habituales de sueño (a), del ajuste entre horas habituales de sueño y horas necesarias de sueño (b), de la latencia de sueño (c), del número de despertares nocturnos (d), de la calidad del sueño: regular o irregular (e) y del grado de satisfacción con la calidad del sueño (f). 
ción típica $=6,79$ ) y, finalmente, las puntuaciones más elevadas en el BDI corresponden a las mujeres con patrón de sueño largo (media $=7,76$; desviación típica $=7,29$ ).

-Ajuste horas habituales de sueño - horas necesarias de sueño

El desajuste entre horas habituales de sueño y horas necesarias de sueño se estableció como la diferencia entre ambas medidas encontrando que 115 sujetos duermen habitualmente menos horas de las que necesitan, 103 sujetos mostraban ajuste, esto es, duermen las horas que necesitan y 39 sujetos duermen habitualmente más horas de las que necesitan. Se han encontrado diferencias significativas $(K-W=6,92, \mathrm{~g} .1 .=2, p<0,05)$ en estado de ánimo disfórico en función del ajuste o no entre horas habituales y horas necesarias de sueño. Comparaciones posteriores indican que las diferencias $(U=$ $4674,00, p<0,01)$ se dan entre los sujetos que duermen habitualmente menos de lo que necesitan y los sujetos que duermen lo que necesitan. En concreto, los sujetos que duermen habitualmente menos de lo que necesitan puntúan más alto en estado de ánimo disfórico (media $=8,44$; desviación típica $=7,11$ ) que los sujetos que duermen lo que necesitan (media $=6,37 ;$ desviación típica $=6,30$ ) (Figura 1b). No se han encontrado diferencias entre hombres y mujeres.

Calidad de sueño y estado de ánimo disfórico

\section{-Latencia de sueño}

Los resultados indican que existen diferencias significativas en ánimo disfórico $(K-W=8,26$, g.l. $=3, p<0,05)$ en función de la latencia de sueño. Las puntuaciones más elevadas en estado de ánimo disfórico corresponden a los sujetos con latencias más prolongadas de sueño (más de 1 hora $)($ media $=11,53$; desviación típica $=$ $7,25)$, seguidos de los sujetos con latencias entre 30 y 60 minutos (media $=7,96$; desviación típica $=7,59$ ), a continuación los sujetos con latencias entre 15 y 30 minutos $($ media $=7,36$; desviación típica $=$ $6,48)$ y, por último, las puntuaciones más bajas corresponden a los sujetos con latencias muy cortas de sueño (menos de 15 minutos) (media $=6,32$; desviación típica $=6,05$ ). Las diferencias significativas se dan entre los intervalos de menos de 15 minutos y más de 1 hora $(U=414,00, p<$ $0,01)$ y los intervalos de entre 15 y 30 minutos y más de 1 hora ( $U=537, p<$ $0,05)$ (Figura 1c). No se han encontrado diferencias entre hombres y mujeres.

\section{—Despertares nocturnos}

Existen diferencias significativas $(K-W=$ $14,92$, g.l. $=2, p<0,01)$ en estado de ánimo disfórico en función del número de despertares nocturnos. Las diferencias significativas se dan entre los sujetos que informan no tener despertares nocturnos nunca o casi nunca y los que suelen despertarse una vez en la noche $(U=2987$, $00, p<0,001)$ y entre los que informan no tener despertares nocturnos nunca o casi nunca y los que suelen despertarse varias veces en la noche $(U=1315,50, p<0,05)$. Concretamente, las puntuaciones medias más elevadas en estado de ánimo disfórico corresponden a los sujetos que se despiertan varias veces en la noche (media $=$ 10,89; desviación típica $=9,31$ ), seguidos de los sujetos que suelen despertarse una vez en la noche (media $=10,26$; desviación típica $=7,53$ ) y, finalmente, las puntuaciones más bajas corresponden a los sujetos que no suelen tener despertares nocturnos nunca o casi nunca (media= 6,32; desviación típica =5,87) (Figura 1d). 
En la muestra de mujeres no se han encontrado diferencias significativas en estado de ánimo disfórico en función del número de despertares nocturnos.

\section{-Profundidad del sueño}

No se han encontrado diferencias significativas en estado de ánimo disfórico en función del grado de profundidad del sueño: ligero, medio o profundo $(K-W=$ 2,50 , g.l. $=2, p=0,28$ ). Esto mismo ocurre cuando se analizan por separado hombres y mujeres.

\section{—Regularidad del sueño}

Se han hallado diferencias significativas $(U=1503,50, p<0,001)$ en estado de ánimo disfórico entre los sujetos en función de la calidad del sueño: regular o irregular. Los sujetos que informan tener un sueño irregular muestran puntuaciones más elevadas en el BDI (media $=11,53$; desviación típica $=8,02$ ) que los sujetos con un sueño regular (media $=6,32$; desviación típica $=5,59$ ) (Figura 1e). No se han encontrado diferencias entre hombres y mujeres.

- Grado de satisfacción con la calidad del sueño

También se han encontrado diferencias significativas en estado de ánimo disfórico en función del grado de satisfacción con la calidad del sueño $(U=4528,00, p$ $<0,001)$. Los sujetos que informan no estar satisfechos con la calidad de su sueño muestran puntuaciones más elevadas en el BDI (media $=10,19$; desviación típi$\mathrm{ca}=7,83$ ) que los sujetos que informan estar satisfechos con la calidad de su sue$\tilde{\text { no }}$ (media $=6,16$; desviación típica $=5,79$ ) (Figura 1f). En la muestra de hombres no se han encontrado diferencias significativas en estado de ánimo disfórico en función del grado de satisfacción con la calidad del sueño.

\section{DISCUSIÓN}

El presente estudio proporciona evidencia de la relación existente entre diversos parámetros subjetivos de sueño y estado de ánimo disfórico. El ánimo disfórico se relaciona tanto con medidas de cantidad como de calidad de sueño. Si bien la relación entre medidas de calidad de sueño y bienestar psicológico se ha encontrado de forma clara en diversos estudios (Hyyppa et al., 1991; Lugaresi et al., 1983; Pilcher et al., 1997), la relación entre duración del sueño y bienestar psicológico no ha sido tan concluyente. Algunos estudios informan que 7-8 horas de sueño a la noche se asocia positivamente con salud autoinformada y longevidad (Belloc y Breslow, 1972; Frederick, Frederichs y Clark, 1988; Kojima et al., 2000; Kripke et al., 2002). Sin embargo, otros no han encontrado diferencias en medidas de salud entre sujetos con patrón de sueño corto y largo (Johns et al., 1974; Spinweber et al., 1985; Webb, 1979).

La cuestión de la duración del sueño y su relación con medidas de estado de ánimo requiere de matizaciones que quizá no se han tenido muy en cuenta en algunos estudios. En el presente estudio se ha comprobado que no son las horas necesarias de sueño lo que determina las diferencias en estado de ánimo sino las horas habituales de sueño. Este tipo de diferenciación es importante si se quiere llegar a un análisis completo de este tema. Concretamente los sujetos que duermen habitualmente 6 horas o menos son los que presentan un peor estado de ánimo. Teniendo en cuenta que lo que duerme habitualmente una persona sue- 
le estar relacionado con condicionantes ambientales, podemos plantearnos que es el desajuste entre las horas necesarias de sueño y las horas habituales de sueño lo que está determinando las diferencias en el estado de ánimo. Efectivamente se han encontrado diferencias en estado de ánimo disfórico en función del ajuste o no entre estos dos parámetros de sueño. Los sujetos que duermen habitualmente menos horas de las que necesitan son los que puntúan más alto en el BDI seguidos de los sujetos que duermen más de lo que necesitan.

En el caso de los sujetos que duermen menos horas de las que necesitan se puede plantear que sea la privación de sueño la explicación más evidente. Muchas personas podrían encontrarse en una situación de privación crónica de sueño que sería lo que estaría afectando a su bienestar psicológico. Existen datos que parecen sugerir que la pérdida de sueño es una pauta habitual en nuestra sociedad. Por ejemplo, la comparación del sueño de los jóvenes de 1963 con los de 1910-1911 mostraba una reducción del tiempo total de sueño de aproximadamente una hora y media, lo que sugiere que tendemos a perder horas de sueño por el actual estilo de vida (Carskadon, 1993). Por otra parte, diversos estudios han señalado los efectos perjudiciales de la privación de sueño en diversas medidas del estado de ánimo (Blagrove y Akehurst, 2001; Brendel et al., 1990; Beutler, Cano, Miró y Buela-Casal, 2003; Cano, Miró y Buela-Casal, 2001; Cutler y Cohen, 1979; Gillberg y Akerstedt, 1981; Hill et al., 1996; Mikulincer et al., 1989; Miró, Cano, Espinosa y Buela-Casal, 2002b; Reynolds et al., 1986).

También se ha encontrado que dormir más horas de las que se necesitan se asocia a un peor estado de ánimo. La explicación de lo que puede estar ocurriendo en estos sujetos es menos obvia. Se sabe que extender el sueño en más horas de lo habitual puede tener efectos perjudiciales sobre el estado de ánimo (Moorcroft, 1993). Diversos autores han asociado algunos de estos efectos a la mayor cantidad de sueño MOR que se obtendría durmiendo más horas. Esta fase de sueño se ha implicado desde el punto de vista funcional en la modulación y regulación del afecto (Kramer, 1993). Otra posibilidad es que pudiera existir una desincronización circadiana en los sujetos con patrón de sueño largo, es decir, algún tipo de alteración en la forma, amplitud y/o posiciones de fase entre diversos ritmos biológicos y el ritmo sueño-vigilia (Miró et al., 2002c).

Por otra parte, se ha encontrado relación entre diversos parámetros de calidad de sueño y estado de ánimo disfórico. Concretamente el ánimo deprimido se relaciona con latencia de sueño, despertares nocturnos, regularidad del sueño y grado de satisfacción con la calidad del sueño. Totterdell et al. (1994) también encuentran que la calidad autoinformada de sueño era el mejor predictor de un buen estado de ánimo al día siguiente. Pilcher et al. (1997) señalan que una pobre calidad de sueño correlaciona significativamente con quejas de salud física y algunas medidas de bienestar psicológico que incluyen incrementos de tensión, depresión, ira, fatiga y confusión.

En cuanto a las diferencias obtenidas en función del género, los resultados señalan que las diferencias entre hombres y mujeres se producen sólo en tres variables: horas necesarias de sueño, número de despertares nocturnos y grado de satisfacción con la calidad del sueño. A diferencia de lo que ocurre en la muestra total y en la muestra de hombres, en la muestra de mujeres se han encontrado diferencias en ánimo disfórico en función de las horas necesarias de sueño. Concretamente las mujeres con patrón de sueño corto obtienen una pun- 
tuación media en el BDI muy baja $(1,67)$ comparadas a las mujeres con patrón de sueño intermedio $(7,48)$ y con patrón de sueño largo $(7,76)$. Sin embargo, conviene señalar que el número de mujeres con patrón de sueño corto es muy pequeño ( $\mathrm{n}$ $=6$ ) como para que estos datos puedan ser considerados concluyentes. Por otra parte, aunque las diferencias en ánimo disfórico en función del número de despertares nocturnos en el grupo de mujeres sigue la misma tendencia que en la muestra total y en la muestra de hombres estas diferencias no llegan a alcanzar significación estadística. Lo mismo ocurre con la variable satisfacción con la calidad del sueño pero en este caso en el grupo de hombres. Aunque los datos señalan la influencia de la variable género en algunos análisis realizados éstos deben ser interpretados con cierta cautela. Como antes apuntábamos el número de hombres o mujeres en algunos grupos es muy reducido. Son necesarios más estudios que analicen a fondo estas diferencias de género.

La duración y calidad de sueño se evaluó en este estudio utilizando un cuestionario elaborado por los propios investigadores del que no se dispone de datos psicométricos lo que puede limitar la generalización de los resultados. El cuestionario además puede tener sesgos de deseabilidad social. No obstante, conviene matizar que la mayor parte de las preguntas incluidas en este cuestionario son las que habitualmente se incluyen en otros cuestionarios sobre hábitos de sueño. Además hemos incorporado otras que han resultado relevantes para hacer un análisis más completo del tema.

No debemos tampoco olvidar que en la relación entre hábitos de sueño y estado de ánimo otras variables pueden estar interviniendo. Puede que la relación entre sueño y bienestar psicológico sea consecuencia de terceras variables. Diversos trabajos se han centrado en el análi- sis de algunas de estas posibles variables (Herrera y Maldonado, 2002; Santos y Bernal, 2001). Son necesarios más estudios longitudinales para conocer la verdadera naturaleza de esta relación.

En suma, los resultados apoyan la importancia del sueño y su relación con medidas de bienestar psicológico en poblaciones no clínicas. Si bien esta relación aparece de forma más intensa en sujetos depresivos los presentes resultados demuestran que la relación aunque más débil se da también en sujetos sanos bajo condiciones normales. Sin embargo, las generalizaciones a muestras clínicas de las conclusiones derivadas de estudios con muestras no clínicas deben ser tomadas siempre con cierta cautela, por lo menos en lo que se refiere a los trastornos emocionales (Ruipérez y Belloch, 1997).

No cabe duda de que el sueño es un excelente indicador del bienestar psicológico tanto de pacientes psiquiátricos o médicos como de la población general. En este sentido es necesario insistir no sólo en la necesidad de tratamientos adecuados para los trastornos del sueño de cara a la prevención de problemas asociados (Echeburúa, 1998) sino también la conveniencia de poner en marcha estrategias preventivas que permitan mejorar nuestros hábitos de sueño.

\section{REFERENCIAS BIBLIOGRÁFICAS}

Akerstedt, T. (1990). Shift work and sleep disturbances. En J.H. Peter, T. Penzel, T. Podszus y P. von Wichert (Eds.), Sleep and health risk (pp. 265-278). Berlin: SpringerVerlag.

Balgrove, M., y Akehurst, L. (2001). Personality and the modulation of effects of sleep loss on mood and cognition. Personality and Individual Differences, 30, 819-828.

Bazargan, M. (1996). Self-reported sleep disturbance among African-american elderly: The effects of depression, health status. 
exercise, and social support. International Journal of Aging and Human Development, 42, 143-160.

Beck, A.T., Rush, A.J., Shaw, B.F., y Emery, G. (1979). Cognitive Therapy of Depression. New York: The Guilford Press.

Beck, A.T., Steer, R.A., y Garbin, M.C. (1988). Psychometric properties of the Beck Depression Inventory: Twenty-five years of evaluation. Clinical Psychology Review, 8, 77-100.

Belloc, N.B., y Breslow, L. (1972). Relationship of physical health status and health practices. Preventive Medicine, 1, 409-421.

Benca, R.M., Obermeyer, W.H., Thisted, R.A., y Gillin, J.C. (1992). Sleep and psychiatric disorders: a meta-analysis. Archives of General Psychiatry, 49, 651-668.

Berry, D.T.R., y Webb, W.B. (1985). Mood and sleep in aging women. Journal of Personality and Social Psychology, 49, 1724-1727.

Beutler, L.E., Cano-Lozano, M.C., Miró, E., y Buela-Casal, G. (2003). The role of activation in the effect of total sleep deprivation on depressed mood. Journal of Clinical Psychology, 59, 369-384.

Brendel, D.H., Reynolds III, C.F., Jennings, F.R., Hoch, C.C., Monk, T.H., Berman, S.R., Hall, F.T., Buysse, D.J., y Kupfer, D.J. (1990). Sleep stage physiology, mood and vigilance responses to total sleep deprivation in healthy 80-year-olds and 20-yearolds. Psychophysiology, 27, 677-685.

Breslau, N., Roth, T., Rosenthal, L., y Andreski, P. (1997). Daytime sleepiness: An epidemiological study of young adults. American Journal of Public Health, 87, 1649-1653.

Bobenrieth, M.A. (2002). Normas para la revisión de artículos originales en Ciencias de la Salud. Revista Internacional de Psicología Clinica/International Journal of Clinical and Health Psychology, 2, 509-523.

Buysse, D.J., Frank, E., Lowe, K.K., Cherry, C.R., y Kupfer, D.J. (1997). Electroencephalographic sleep correlates of episode and vulnerability to recurrence in depression. Biological Psychiatry, 41, 406-418.

Cano, M.C., Espinosa, L., Miró, E., y BuelaCasal, G. (2003). Una revisión de las alteraciones del sueño en la depresión. Revista de Neurología, 36, 366-375.
Cano, M.C., Miró, E., y Buela-Casal, G. (2001). Efecto de la privación total de sueño sobre el estado de ánimo disfórico y su relación con los cambios en la activación autoinformada. Salud Mental, 24, 41-50.

Carroll, B.J., Fielding, J.M., y Blashky, T.G. (1973). «Depression rating scales». Archives of General Psychiatry, 28, 361-366.

Carskadon, M. A. (1993). Encyclopedia of Sleep and Dreaming. New York: Macmillian.

Cutler, N.R., y Cohen, H.B. (1979). The effect of one night's sleep loss on mood and memory in normal subjects. Comprensive Psychiatry, 20, 61-66.

Dew, M.A., Reynolds, C.F., Buysse, D.J., Houck, P.R., Hoch, C.C., Monk, T.H., y Kupfer, D.J. (1996). Electroencephalographic sleep profiles during depression. Archives of General Psychiatry, 53, 148156.

Dinges, D.F., Pack, F., Williams, K., Gillen, K.A., Powell, J.W., Ott, G.E., Aptowicz, C., y Pack, A.I. (1997). Cumulative sleepiness, mood disturbances and psychomotor vigilance performance decrements during a week of sleep restricted to 4-5 hours per night. Sleep, 20, 267-277.

Duncan, D.F., Bomar, G.J., Nicholson, T., y Wilson, R. (1995). Health practices and mental health revisited. Psychological Reports, 77, 205-206.

Echeburúa, E. (1998). ¿Qué terapias de conducta son eficaces? Un reto ante el año 2000. Revista de Psicopatología y Psicología Clínica, 3, 149-160.

Ford, D.E., y Kamerow, D.B. (1989). Epidemiologic study of sleep disturbances and psychiatric disorders: an opportunity for prevention? JAMA, 262, 1479-1484.

Frederick, T., Frederichs, R.R., y Clark, V.A. (1988). Personal health habits and symptoms of depression at the community level. Preventive Medicine, 17, 173-182.

Gillberg, M., y Akerstedt, T. (1981). Sleep deprivation in normals-some psychological and biochemical data from three studies. En W.P. Koella (Ed.), Sleep 1980 (pp. 16-22). Amsterdam: Karger, Basel.

Gillin, J.C., Sitaram, N., Wehr, T., Duncan, W.C., Post, R.M., Murphy, D.L., Mendelson, W.B., Wyatt, R.J., y Bunney. W.E. 
(1984). Sleep and affective illness. En R.M. Post y J.C. Ballenger (Eds.), Neurobiology of mood disorders (pp. 157-188). Baltimore: Williams \& Wilkins.

Habte-Gabr, E., Wallace, R.B., Colsher, P.L., Hulbert, J.R., White, L.R., y Smith, I.M. (1991). Sleep patterns in rural elders: Demographic, health, and psychobehavioral correlates. Journal of Clinical Epidemiology, 44, 5-13.

Herrera, A., y Maldonado, A. (2002). Depresión, cognición y fracaso académico. Revista Internacional de Psicología Clínica y de la Salud/International Journal of Clinical and Health Psychology, 2, 25-50.

Hill, D.W., Welch, J.E., y Godfrey III, J.A. (1996). Influence of locus of control on mood state disturbance after short-term sleep deprivation. Sleep, 19, 41-46.

Hyyppa, M.T., Kronholm, E., Mattlar, C.E. (1991). Mental well-being of good sleepers in a random population sample. British Journal of Medical Psychology, 64, 25-34. Johns, M.W., Bruce, D.W., y Masterton, J.P. (1974). Psychological correlates of sleep habits reported by healthy young adults. British Journal of Medical Psychology, 47, 181-187.

Kojima, M., Wakai, K., Kawamura, T., Tamakoshi, A., Aoki, R., Lin, Y., Nakayama, T., Horibe, H., Aoki, N., y Ohno, Y. (2000). Sleep patterns and total mortality: A 12 year follow-up study in Japan. Journal of Epidemiology, 10, 87-93.

Kramer, M. (1993). The selective mood regulatory function of dreaming: And update and revision. En A. Moffitt, M. Kramer y R. Hoffmann (Dirs.), The functions of dreaming (pp. 139-195). New York: State University of New York Press.

Kripke, D.F., Garfinkel, L., Wingard, D., Klauber, M.R., y Marter, M.R. (2002). Mortality associated with sleep duration and insomnia. Archives of General Psychiatry, 59, 131-136.

Kupfer, D.J. (1995). Sleep research in depressive illness: Clinical Implications- A tasting menu. Biological Psychiatry, 38, 391-403.

Leproult, R., Colecchia, E.F., Berardi, A.M., Stickgold, R., Kosslyn, S.M., y Van Cauter, E. (2003). Individual differences in subjective and objective alertness during sleep deprivation are stable and unrelated. American Journal of Physiology Regulatory, Integrative and Comparative Physiology, 284, R 280-290.

Lugaresi, E., Cirignotta, F., Zucconi, M., Mondini, S., Lenzi, P.L., y Coccagna, G. (1983). Good and poor sleepers: an epidemiological survey of the San Marino population. En C. Guilleminault y E. Lugaresi (Eds.), Sleep/wake disorders. Natural history, epidemiology and long-term evolution (pp. 13-28). New York: Raven Press.

Mahon, N.E. (1995). The contributions of sleep to perceived health status during adolescence. Public Health Nursing, 12, 127 133.

Matousek, M., Nuth, A.L., y Petersen, I. (1983). Spontaneous vigilance fluctuations in the daytime as reflected by EEG and psychological variables. Advances in Biological Psychiatry, 13, 13-18.

Mikulincer, M., Babkoff, H., Caspy, T., y Sing, H. (1989). The effects of 72 hours of sleep loss on psychological variables. British Journal of Psychology, 80, 145-162.

Miró, E., Cano, M.C., y Buela-Casal, G. (2002a). Electrodermal activity during total sleep deprivation and its relationship with others activation and performance measures. Journal of Sleep Research, 11, 105-112.

Miró, E., Cano, M.C., Espinosa, L., y BuelaCasal, G. (2002b). Análisis de la relación entre género y respuestas de activación. estrés y ansiedad ante una situación de privación prolongada de sueño. Ansiedad y Estrés, 8, 193-209.

Miró, E., Iáñez, M.A., y Cano, M.C. (2002c). Patrones de sueño y salud. Revista Internacional de Psicología Clinica/International Journal of Clinical and Health Psychology, 2, 301-326.

Montero, I., y León, O. (2002). Clasificación y descripción de las metodologías de investigación en Psicología. Revista Internacional de Psicología Clinica/International Journal of Clinical and Health Psychology, 2, 503-508.

Moorcroft, W.H. (1993). Sleep, Dreaming and Sleep Disorders. Boston: University Press of America.

Ohayon, N.M., Priest, R.G., Zulley, J., y Smirne, S. (2000). The place of confusional 
arousals in sleep and mental disorders finding in a general population sample of 13.057 subjects. Journal of Nervous and Mental Disease, 188, 340-348.

Oullet, M.N. (1995). Sleep satisfaction of older adults living in the community and related factors. Case Western Reserve University, 216.

Pilcher, J. J., Ginter, D.R., y Sadowsky, B. (1997). Sleep quality versus sleep quantity: Relationships between sleep and measures of health, well-being and sleepiness in college students. Journal of Psychosomatic Research, 42, 583-598.

Regestein, Q.R., y Monk, TH. (1991). Is the poor sleep of shift workers a disorder? American Journal of Psychiatry, 148, 1487 1493.

Reynolds III, C.F., Kupfer, D.J., Hoch, C.C., Stack, J.A., Houck, P.R., y Berman, S.R. (1986). Sleep deprivation in healthy elderly men and women: Effects on mood and on sleep during recovery. Sleep, 9 , 492-501.

Riemann, D., Berger, M., y Voderholzer, U. (2001). Sleep and depression-results from psychobiological studies: an overview. Biological Psychology, 57, 67-103.

Ruipérez, M.A., y Belloch, A. (1997). Depresión y autoesquemas depresivos en pacientes deprimidos y ansiosos. Revista de Psicopatología y Psicología Clínica, 2, 65-80.
Santos, A., y Bernal, G. (2001). Eventos estresantes vitales y sintomatología depresiva en tres muestras puertorriqueñas. Revista Internacional de Psicología Clínica y de la Salud/International Journal of Clinical and Health Psychology, 1, 475-494.

Shaver, J.L.F., Giblin, E., y Paulsen, V. (1991). Sleep quality subtypes in midlife women. Sleep, 14, 18-23.

Spinweber, C.L., Johson, L.C., y Chin, L.A. (1985). Disqualified and qualified poor sleepers: subjective and objective variables. Health Psychology, 4, 569-578.

Steer, R.A., Beck, A.T., y Garrison, B. (1986). Applications of the Beck Depression Inventory. En N. Sartorius y T.A. Ban (Eds.), Assessment of depression (pp. 121142). Berlin: Springer-Verlag.

Totterdell, P, Reynolds, S., Parkinson, B., y Briner, R.B. (1994). Associations of sleep with everyday mood, minor symptoms and social interaction experience. Sleep, 17, 466-475.

Vázquez, C., y Sanz, J. (1991). Fiabilidad y validez factorial de la versión española del Inventario de Depresión de Beck. Comunicación presentada en el III Congreso de Evaluación Psicológica, Barcelona, 25-28 de septiembre.

Webb, W.B. (1979). Are short and long sleepers different? Psychological Reports, 44, 259-264. 